\title{
Diversidad de grupos funcionales en la vegetación de la costa arenosa de los cayos Grande, Anclitas y Caguamas (archipiélago de los Jardines de la Reina, Cuba)
}

\author{
Carlos J. Acevedo Rodríguez ${ }^{1}$ y Luis Roberto González-Torres²
}

\begin{abstract}
Resumen: Acevedo Rodríguez, C. J. \& González-Torres, L. R. 2015. Diversidad de grupos funcionales en la vegetación de la costa arenosa de los cayos Grande, Anclitas y Caguamas (archipiélago de los Jardines de la Reina, Cuba). Bot. Complut. 39: 97-104.

Las costas arenosas son ambientes extremos donde la vegetación desarrolla adaptaciones morfológicas y fisiológicas para su supervivencia. Con la finalidad de cuantificar y relacionar la diversidad funcional con factores abióticos, se tomaron datos de riqueza y abundancia en la vegetación de costa arenosa de los cayos Grande, Anclitas y Caguamas. La flora se caracteriza por poseer un predominio de plantas caméfitas y hemicriptófitas, con hojas nanófilas y síndromes de dispersión de anemocoria y zoocoria. No obstante, la riqueza de los grupos funcionales varía entre los cayos estudiados. La diversidad funcional es semejante entre las estaciones lluvia y seca, y su variación espacial depende de la interacción del conjunto de factores abióticos medidos.
\end{abstract}

Palabras clave: archipiélago, cayos, diversidad, grupos funcionales, vegetación.

Abstract: Acevedo Rodríguez, C. J. \& González-Torres, L. R. 2015. Diversity of functional groups in the vegetation sandy seashore of the Grande, Anclitas and Caguamas Cays (Jardines de la Reina Archipelago, Cuba). Bot. Complut. 39: 97-104.

Sandy shores are known to be extreme ecosystems where the vegetation has evolved many morphological and physiological adaptations for its survival. With the aim of identify possible relationships between the vegetation's functional diversity with abiotic factors and its corresponding quantification, we collected data on the abundance and richness of the sandy coast vegetation complex in Grande, Anclitas and Caguamas keys. Its flora is largely characterized by the dominance of hemicryptophytes and chamaephytes plants with nanophyllous leaves and displaying dispersal syndromes such as zoochory and anemochory. However, the functional groups' richness, in the present study, varies from one key to another. Functional diversity is similar between the wet and dry seasons, and its spatial variation is influenced by the interplay of the set of abiotic factors herein studied.

Key words: archipelago, keys, diversity, functional groups, vegetation.

\section{INTRODUCCIÓN}

En las costas arenosas se establecen plantas con características particulares, que tienen que afrontar un ambiente extremo durante su desarrollo (Águila et al. 1995). Las condiciones edáficas y la proximidad al mar, hacen que solo un número limitado de especies vegetales sean capaces de vivir en estas condiciones ambientales. Esto hace que la flora de las costas arenosas sea relativamente uniforme y diferente respecto a otros ecosistemas terrestres. Esencialmente la vegetación de costa arenosa, está constituida por especies psammófilas, xerófilas, halófilas y oligótrofas, resistentes al viento y a la movilidad del substrato (García-Albá \& Morey 1981). Según Hesp (1991), las plantas que crecen en los ecosistemas dunares han desarrollado adaptaciones para tolerar altos contenidos de sal. Algunos autores, han realizado experimentos con la finalidad de conocer la resistencia de las especies vegetales a las elevadas concentraciones de sal, lo cual constituye una herramienta importante para futuros planes de restauración ecológica (Tiku \& Snaydon 1971, Woodel 1985, Blits \& Gallagher 1991, Martínez et al. 1992).

Debido a la naturaleza arenosa que presenta el substrato en las dunas costeras, existe una baja retención de agua (Martínez et al. 1992). Se considera que los bajos potenciales hídricos no afectan a las especies vegetales que

\footnotetext{
${ }^{1}$ Facultad de Ciencias Agropecuarias, Universidad Máximo Gómez Báez, Carretera a Morón, Km 9, 69450-Provincia Ciego de Ávila, Cuba.carlosj@sma.unica.cu

${ }^{2}$ Iniciativa Planta, Sociedad Cubana de Botánica.

Recibido: 26 septiembre 2014. Aceptado: 13 febrero 2015.
} 
habitan las costas arenosas, pues muchas de estas han desarrollado adaptaciones morfológicas y fisiológicas que le permiten sobrevivir ante condiciones extremas de sequía (Hesp 1991). Entre las principales adaptaciones de las especies vegetales que habitan en las costas arenosas se encuentran: el ajuste osmótico, presencia de estructuras xeroformas, enrollamientos de hojas, pubescencia, secreción de ceras, semillas con testa dura, germinación diferida, presencia de un porte terófito y hemicriptófitos, etc. (Barbour et al. 1985, Hesp 1991, San Martín et al. 1992).

La estacionalidad se encuentra muy ligada a los hábitos o formas de vida de algunas especies vegetales. San Martín et al. (1992) refieren la existencia de mayor número de especies terófitas y hemicriptófitas que soportan valores extremos de sequía y baja pluviosidad. El predominio de estas formas de vida dentro de una comunidad vegetal puede establecer diferencias en la composición de especies entre los meses de lluvia y seca. Por otra parte, la zonación de las dunas costeras se encuentra estrechamente relacionada con la vegetación. Por ejemplo, las especies vegetales que colonizan la zona pionera están adaptadas a inundaciones por la subida del nivel del mar, aspersión salina, sequía, calor y bajo suministro de nu- trientes (Moreno-Casasola 1982, Williams 2007). Algunas de estas plantas han desarrollado densos sistemas radiculares y elevada producción de flores y semillas (Williams 2007).

La biodiversidad generalmente ha sido expresada por índices que utilizan los criterios de riqueza y abundancia de las especies (Shannon-Weaver 1949, Simpson 1949). Sin embargo, recientemente ha sido empleado el término de diversidad funcional (Schleuter et al. 2010), el cual caracteriza a la comunidad a partir de grupos funcionales (Poos et al. 2009). En este trabajo se cuantifican los grupos funcionales de las especies vegetales que integran a la vegetación de costa arenosa de los cayos Grande, Anclitas y Caguamas, archipiélago de los Jardines de la Reina; así como su variación y relación con factores abióticos.

\section{MATERIALES Y MÉTODOS}

Los cayos, Grande, Anclitas y Caguamas, forman parte del grupo insular Jardines de la Reina. Este archipiélago se localiza en la parte meridional de la isla de Cuba, al sur de las provincias de Sancti Spíritus, Ciego de Ávila y Camagüey (Fig. 1). Su origen geológico se remonta a la última glaciación del Pleis-

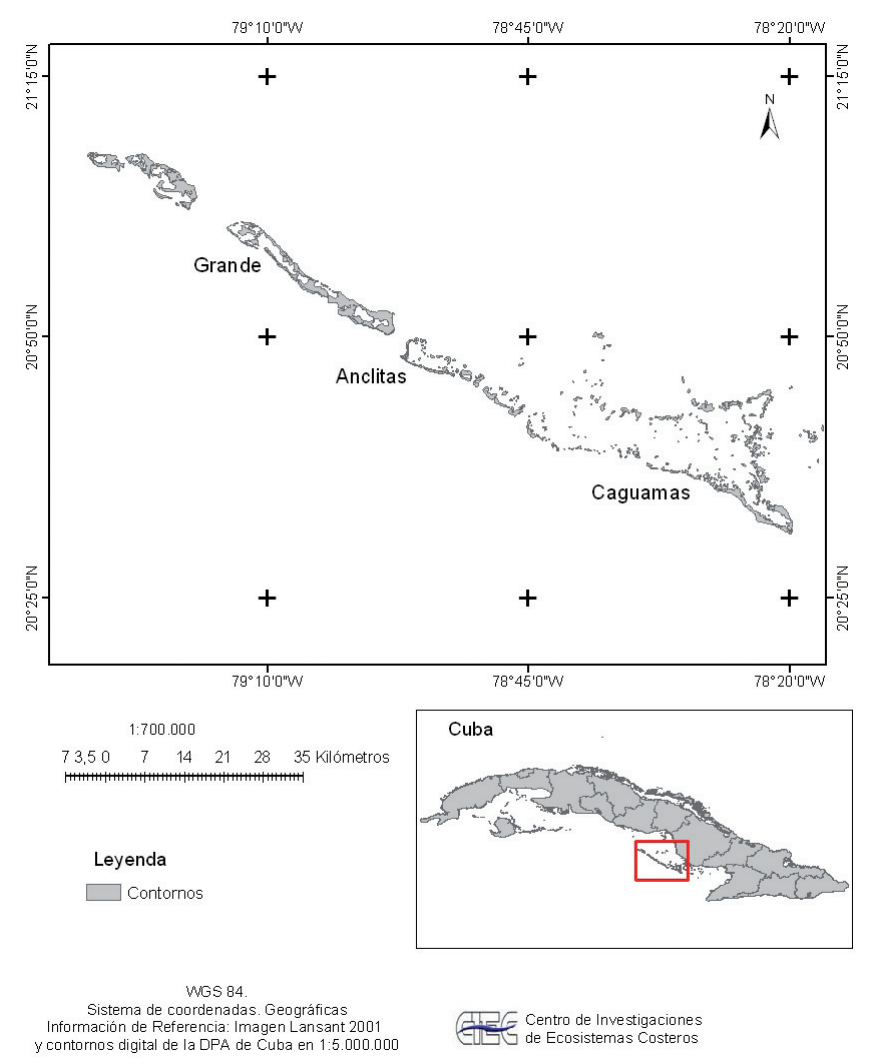

Fig. 1- Localización de los cayos Grande, Anclitas y Caguamas, archipiélago de los Jardines de la Reina, Cuba. 
toceno, donde se formaron las rocas biocalcarenitas. Dichas rocas, conjuntamente con el proceso de su meteorización y el aporte de sedimento arenoso durante el Holoceno, dieron lugar a espacios semiáridos, en los cuales se formaron las dunas y los camellones de tormenta presente en el sitio (Zúñiga 2000). De acuerdo a la regionalización climática de Díaz (1989), el área de estudio se encuentra dentro del tipo de llanuras costeras y cayos con humedecimiento insuficiente, muy alta evaporación y temperaturas cálidas que oscilan entre 24 y $31^{\circ} \mathrm{C}$ (González de Zayas et al. 2006).

En cada cayo se establecieron 20 parcelas permanentes distribuidas al azar, con dimensiones de $2 \times 2 \mathrm{~m}$, donde se tomaron datos de composición de especie y número de individuos. La lista florística se realizó a partir de la identificación in situ, la comparación de los materiales recolectados con ejemplares procedentes del herbario HAJB y la consulta de la literatura (León 1946; León \& Alain 1951; Alain 1953, 1957, 1964; Zona 1990; Rodríguez 2000 y Catasús 2002). Los nombres de las especies se actualizaron según Acevedo-Rodríguez \& Strong (2012). Los datos de abundancia se obtuvieron por especies; en el caso de las gramíneas y ciperáceas cada clon fue considerado como un individuo.

Para la clasificación de los taxones en grupos funcionales, se usaron los criterios de resistencia de las plantas a épocas desfavorables o tipo biológico (Raunkiaer 1934) y mecanismos de dispersión (van der Pijl 1982). En el desarrollo vegetativo de la planta o tamaño de la hoja, se empleó el criterio de Borhidi (1976). Dicha clasificación es empleada para los estudios florísticos de la vegetación cubana y comprende los siguientes caracteres funcionales: leptófila $\left(>25 \mathrm{~mm}^{2}\right)$, nanófila $(25-225$ $\left.\mathrm{mm}^{2}\right)$, micrófila $\left(225-2,025 \mathrm{~mm}^{2}\right)$, notófila $\left(2,025-4,500 \mathrm{~mm}^{2}\right.$, mesófila $\left(4,500-18,225 \mathrm{~mm}^{2}\right)$, macrófila $\left(18,225-164,025 \mathrm{~mm}^{2}\right)$ y megáfila $\left(<\right.$ de $\left.164,025 \mathrm{~mm}^{2}\right)$.

El hábito y mecanismo de dispersión se definió mediante la literatura usada para la identificación taxonómica, en el caso de los mecanismos de dispersión fueron consultados además Catasús (1997), Lonard et al. (2010), Skaradek \& Miller (2010) y GISD (2012). Los datos de composición y número de individuos fueron tomados durante los días 14-22 de abril y el 17-26 de octubre de 2010, coincidiendo estos con los períodos de seca y lluvia respectivamente. En cada unidad de muestreo fueron tomados además, datos abióticos (distancia de la parcela a la línea de costa, altura de la duna y granulometría del substrato) según criterios de Acevedo \& González-Torres (2013).

Mediante datos de riqueza observada por parcelas de cada carácter funcional, se cuantificó el promedio de los mismos para cada cayo. La diversidad funcional fue determinada para ambas estaciones del año (seca y lluvia) a partir de la expresión matemática propuesta por Kader \& Perry (2007). Cuya fórmula es $\mathrm{FD}_{\text {cat }}=1-\Sigma\left(\mathrm{A}_{l} / \mathrm{A}\right)^{2}, \mathrm{~A}_{l}$ representa la abundancia de especies en la parcela, que posean un carácter funcional (e.g abundancia de especies con hojas nanófilas) y A, abundancia total de especies en el cayo. Para comparar la diversidad funcional entre épocas se utilizó la prueba estadística t de Student, la cual permite la comparación de dos medias cuando estas cumplen con la normalidad y homogeneidad de varianza (StatSoft Inc.
2001). El promedio de la diversidad funcional, se obtuvo a partir de los valores calculados por estaciones (seca y lluvia), este fue empleado para determinar la influencia de las variables abióticas a partir del análisis multivariado de regresión múltiple (StatSoft Inc. 2001).

\section{RESULTADOS}

La flora vascular de los cayos estudiados está constituida por 21 especies, las cuales se agrupan en igual número de géneros y en 11 familias botánicas, siendo Poaceae la de mayor representatividad (Tabla 1). El endemismo es bajo y solamente se encuentra representado por Coccothrinax littoralis. El número total de taxones por localidades se comporta de la siguiente forma: 14 en Cayo Caguamas, 11 en Cayo Anclitas y 8 en Cayo Grande. C. littoralis constituye la especie de mayor abundancia en Cayo Grande $(4,15)$, mientras que Erithalis fruticosa $(10,25)$ y Schizachyrium gracile $(9,85)$ lo son para Anclitas. E. fruticosa $(4,7)$ también resulta el taxón de mayor abundancia en Caguamas. Las especies comunes para los tres cayos son: $C$. littoralis, $E$. fruticosa, $S$. gracile y Rhynchospora sp. Mientras que Corchorus hirsutus y Strumpfia maritima solamente se registran en Cayo Grande, de igual forma sucede con Conocarpus erectus, Chamaecrista lineata y Ernodea littoralis en Cayo Anclitas. Caguamas resulta la localidad de mayor número de especies exclusivas (8 taxones), destacándose la familia Poaceae (6 especies).

Los tipos biológicos predominantes son los caméfitos (7) y hemicriptófitos (8), representando el 33 y $38 \%$ respectivamente del total. En cuanto al tamaño de la hoja, las nanófilas ocupan la primera posición (13) con un $62 \%$. $\mathrm{La}$ anemocoria y zoocoria constituyen los síndromes de dispersión con mayores valores de riqueza absoluta (10 y 8 respectivamente) y estos representan el 47 y $38 \%$ (Tabla 1). El 75\% de los caméfitos poseen hojas nanófilas y dispersión por zoocoria. Mientras que el $100 \%$ de los hemicriptófitos presentan hojas nanófilas y el $88 \%$ dispersión por anemocoria. Las especies Euphorbia thymifolia y Rhynchospora sp. clasificadas como terófitas, se componen de hojas leptófilas y utilizan el viento para su diseminación (anemocoria).

En los cayos Grande y Anclitas el grupo funcional de mayor representatividad de acuerdo a la resistencia a épocas desfavorables es el caméfito, con valores promedio de 1,28 en cayo Grande y 1,65 en cayo Anclitas (Fig. 2). Sin embargo, en cayo Caguamas el mayor promedio de riqueza media es observado en hemicriptófitas con 1,18 (Fig. 2). 
Tabla 1

Lista de grupos funcionales en la costa arenosa de cayo Grande, Anclitas y Caguamas, archipiélago de los Jardines de la Reina, Cuba. 1: Alain (1953), 2: Alain (1957), 3: Alain (1964), 4: Catasús (1997), 5: Catasús (2002), 6: GISD (2012), 7: León (1946), 8: León \& Alain (1951), 9: Lonard et al. (2010), 10: Rodríguez (2000), 11: Skaradek \& Miller (2010), 12: Zona (1990)

\begin{tabular}{|c|c|c|c|c|c|c|c|}
\hline \multirow{2}{*}{ Especies } & \multirow{2}{*}{ Familia } & \multicolumn{3}{|c|}{ Cayos } & \multirow{2}{*}{$\begin{array}{c}\text { Tipo } \\
\text { biológico }\end{array}$} & \multirow{2}{*}{$\begin{array}{l}\text { Tamaño } \\
\text { hoja }\end{array}$} & \multirow{2}{*}{$\begin{array}{l}\text { Mecanismo } \\
\text { dispersión }\end{array}$} \\
\hline & & Grande & Anclitas & Caguamas & & & \\
\hline Chamaecrista lineata (Sw.) Greene & Fabaceae & $x$ & & & Caméfitos ${ }^{(8)}$ & Nanófila & Zoocoria $^{(8)}$ \\
\hline Coccothrinax littoralis León & Arecaceae & $x$ & $x$ & $x$ & Fanerófito ${ }^{(7)}$ & Megáfila & Zoocoria ${ }^{(7)}$ \\
\hline Conocarpus erectus L. & Combretaceae & & $x$ & & Fanerófito ${ }^{(1)}$ & Micrófila & Hidrocoria $^{(1)}$ \\
\hline Corchorus hirsutus L. & Malvaceae & $x$ & & & Caméfitos $\left({ }^{10)}\right.$ & Nanófila & Anemocoria ${ }^{(10)}$ \\
\hline Crossopetalum rhacoma Crantz & Celastraceae & $x$ & $x$ & $x$ & Caméfitos ${ }^{(1)}$ & Nanófila & Zoocoria $^{(1)}$ \\
\hline Distichlis spicata (L.) Greene & Poaceae & & & $x$ & Hemicriptófito ${ }^{(7)}$ & Nanófila & Anemocoria $^{(11)}$ \\
\hline Erithalis fruticosa L. & Rubiaceae & $x$ & $x$ & $x$ & Caméfitos ${ }^{(3)}$ & Micrófila & Zoocoria $^{(3)}$ \\
\hline Ernodea littoralis Sw. & Rubiaceae & & $x$ & & Caméfitos ${ }^{(3)}$ & Nanófila & Zoocoria $^{(3)}$ \\
\hline Euphorbia thymifolia L. & Euphorbiaceae & & $x$ & $x$ & Terófito ${ }^{(1)}$ & Leptófila & Anemocoria ${ }^{(1)}$ \\
\hline Eustachys petraea (Sw.) Desv. & Poaceae & & & $x$ & Hemicriptófito ${ }^{(7)}$ & Nanófila & Anemocoria ${ }^{(7)}$ \\
\hline Fimbristylis cymosa $\mathrm{R} . \mathrm{Br}$. & Cyperaceae & & & $x$ & Hemicriptófito ${ }^{(7)}$ & Nanófila & Anemocoria ${ }^{(7)}$ \\
\hline Metopium toxiferum (L.) Krug \& Urb. & Anacardiaceae & $x$ & $x$ & & Fanerófito ${ }^{(1)}$ & Notófila & Zoocoria $^{(1)}$ \\
\hline Muhlenbergia capillaris (Lam.) Trin. & Poaceae & & & $x$ & Hemicriptófito ${ }^{(7)}$ & Nanófila & Anemocoria ${ }^{(4)}$ \\
\hline Paspalum vaginatum $\mathrm{Sw}$. & Poaceae & & & $x$ & Hemicriptófito ${ }^{(7)}$ & Nanófila & Anemocoria ${ }^{(5)}$ \\
\hline Rhynchospora sp. & Cyperaceae & $x$ & $x$ & $x$ & Terófito ${ }^{(7)}$ & Leptófila & Anemocoria ${ }^{(7)}$ \\
\hline Sabal maritima (Kunth) Burret & Arecaceae & & & $x$ & Fanerófito ${ }^{(12)}$ & Megáfila & Zoocoria $^{(12)}$ \\
\hline Schizachyrium gracile (Spreng.) Nash & Poaceae & $x$ & $x$ & $x$ & Hemicriptófito $^{(7)}$ & Nanófila & Anemocoria ${ }^{(5)}$ \\
\hline Spartina patens (Aiton) Muhl. & Poaceae & & & $x$ & Hemicriptófito ${ }^{(7)}$ & Nanófila & Hidrocoria ${ }^{(9)}$ \\
\hline Sporobolus indicus (L.). R. Br. & Poaceae & & & $x$ & Hemicriptófito $^{(7)}$ & Nanófila & Anemocoria ${ }^{(6)}$ \\
\hline Strumpfia maritima Jacq. & Rubiaceae & $x$ & & & Caméfitos ${ }^{(3)}$ & Nanófila & Zoocoria $^{(3)}$ \\
\hline Tournefortia gnaphalodes (L.) R. Br. & Boraginaceae & & $x$ & $x$ & Caméfitos ${ }^{(2)}$ & Micrófila & Hidrocoria ${ }^{(2)}$ \\
\hline
\end{tabular}

Por otra parte, en los tres cayos estudiados el tipo de hoja más representativo es la nanófila, aunque en cayo Anclitas también resulta mayor el promedio de especies con ho-

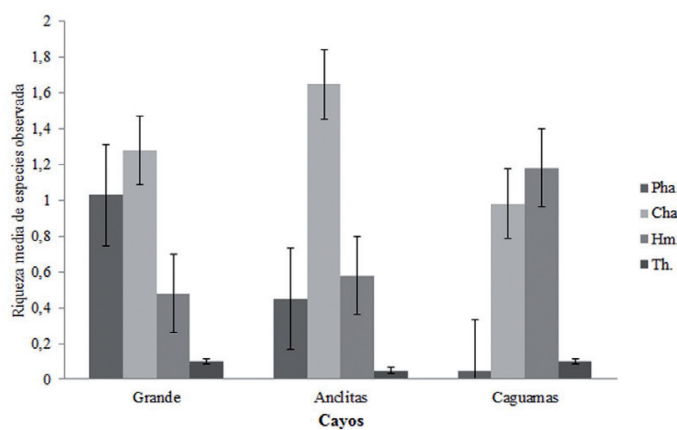

Fig. 2- Espectro biológico en función a la resistencia de las plantas a épocas desfavorables o tipo biológico, cayos Grande, Anclitas y Caguamas, archipiélago de los Jardines de la Reina, Cuba. Pha: Fanerófito, Ch: Caméfito, Hm: Hemicriptófito, Th: Terófito. jas micrófilas (Fig. 3). En los cayos Grande y Anclitas la mayor parte de las especies poseen zoocoria como mecanismo de dispersión, 2,03 y 1,95 respectivamente (Fig. 4).

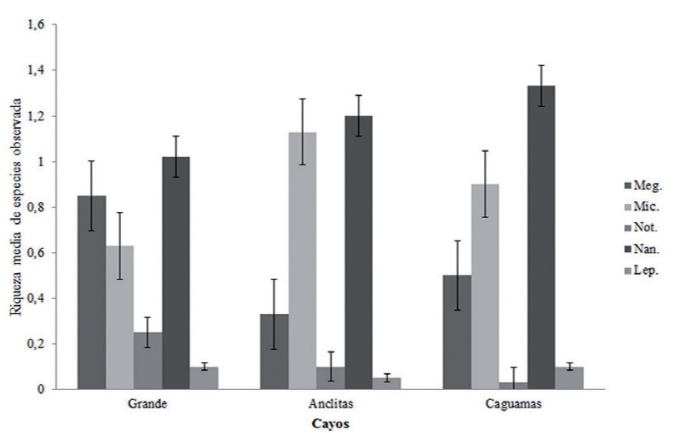

Fig. 3- Espectro biológico en función al desarrollo vegetativo de la planta o tamaño de la hoja, cayos Grande, Anclitas y Caguamas, archipiélago de los Jardines de la Reina, Cuba. Meg: Megáfila, Mic: Micrófila, Not: Notófila, Nan: Nanófila, Lep: Leptófila. 


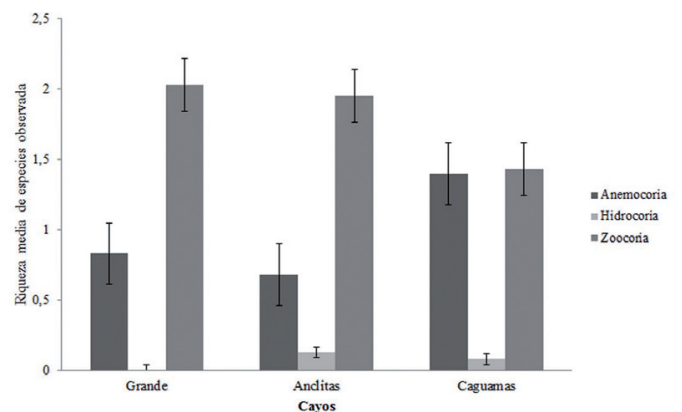

Fig. 4- Espectro biológico en función a los mecanismos de dispersión de las plantas, cayos Grande, Anclitas y Caguamas, archipiélago de los Jardines de la Reina, Cuba.

En cayo Caguamas los síndromes más comunes son zoocoria $(1,43)$ y anemocoria $(1,40)$ (Fig. 4).
En los cayos Grande, Anclitas y Caguamas la diversidad funcional es baja, con valores de 0,997-0,998 y esta no varía entre cayos ni épocas (Tabla 2). En el análisis de regresión múltiple, las variables abióticas con relación a la diversidad funcional presentan un coeficiente, con valores cercanos a cero y $p>0,05$. Esto indica que existe una baja contribución de las variables ábióticas sobre la diversidad funcional (Tablas 3, 4 y 5).

\section{DISCUSIÓN}

Los resultados obtenidos en cuanto a tipo biológico no coinciden con los expuestos por Álvarez \& Ricardo (2009) donde estos listaron un mayor número de terófitas en las dunas de Playa del Este, La Habana. El elevado nú-

Tabla 2

Variación de la diversidad funcional durante el período seco (14-22 de abril de 2010) y el período lluvioso (17-26 de octubre de 2010) en los cayos Grande, Anclitas y Caguamas, archipiélago de los Jardines de la Reina, Cuba

\begin{tabular}{|c|c|c|c|c|c|c|}
\hline \multirow{2}{*}{ Localidades } & \multirow{2}{*}{ Grupos funcionales } & \multicolumn{2}{|c|}{ Media \pm Desviación Estándar } & \multirow{2}{*}{$\mathrm{t}$} & \multirow{2}{*}{ d.f. } & \multirow{2}{*}{$\mathrm{p}$} \\
\hline & & Seca & Lluvia & & & \\
\hline \multirow[t]{3}{*}{ Grande } & Tipo biológico & $0,998 \pm 0,003$ & $0,998 \pm 0,001$ & $-0,339$ & 38 & 0,735 \\
\hline & Tipo de hoja & $0,997 \pm 0,003$ & $0,997 \pm 0,001$ & $-0,432$ & 38 & 0,667 \\
\hline & Mecanismo de dispersión & $0,997 \pm 0,004$ & $0,997 \pm 0,002$ & $-0,168$ & 38 & 0,866 \\
\hline \multirow[t]{3}{*}{ Anclitas } & Tipo biológico & $0,997 \pm 0,002$ & $0,997 \pm 0,003$ & 0,015 & 38 & 0,998 \\
\hline & Tipo de hoja & $0,998 \pm 0,002$ & $0,998 \pm 0,003$ & 0,031 & 38 & 0,975 \\
\hline & Mecanismo de dispersión & $0,998 \pm 0,002$ & $0,997 \pm 0,006$ & 0,401 & 38 & 0,690 \\
\hline \multirow[t]{3}{*}{ Caguamas } & Tipo biológico & $0,998 \pm 0,002$ & $0,998 \pm 0,002$ & $-0,260$ & 38 & 0,796 \\
\hline & Tipo de hoja & $0,998 \pm 0,002$ & $0,998 \pm 0,002$ & $-0,439$ & 38 & 0,662 \\
\hline & Mecanismo de dispersión & $0,998 \pm 0,002$ & $0,998 \pm 0,002$ & 0,212 & 38 & 0,833 \\
\hline
\end{tabular}

Tabla 3

Relación de la diversidad funcional (resistencia a épocas desfavorables o tipo biológico) con las variables abióticas medidas

\begin{tabular}{llrrrrrc}
\hline Cayos & \multicolumn{1}{c}{ Variables } & Beta & Err Std. & B & Err. Std. & t(16) & p \\
\hline Grande & Dist. a línea de costa & $-0,402378$ & 0,217028 & $-0,000057$ & 0,000031 & $-1,8540$ & 0,082262 \\
& Altura de la duna & 0,226160 & 0,218932 & 0,001067 & 0,001033 & 1,0330 & 0,316959 \\
& Granulometría & $-0,235600$ & 0,216873 & $-0,007282$ & 0,006703 & $-1,0864$ & 0,293420 \\
\multirow{4}{*}{ Anclitas } & Dist. a línea de costa & $-0,122525$ & 0,253729 & $-0,000018$ & 0,000038 & $-0,4829$ & 0,635711 \\
& Altura de la duna & $-0,105675$ & 0,271487 & $-0,000131$ & 0,000337 & $-0,3892$ & 0,702226 \\
& Granulometría & 0,160862 & 0,259345 & 0,002446 & 0,003944 & 0,6203 & 0,543819 \\
Caguamas & Dist. a línea de costa & 0,259091 & 0,242234 & 0,000024 & 0,000022 & 1,0696 & 0,300675 \\
& Altura de la duna & $-0,317489$ & 0,239565 & $-0,002479$ & 0,001871 & $-1,3253$ & 0,203695 \\
& Granulometría & $-0,124753$ & 0,237068 & $-0,002953$ & 0,005613 & $-0,5262$ & 0,605947 \\
\hline
\end{tabular}


Tabla 4

Relación de la diversidad funcional (desarrollo vegetativo o tamaño de la hoja) con las variables abióticas medidas

\begin{tabular}{llrrrrrr}
\hline Cayos & \multicolumn{1}{c}{ Variables } & Beta & Err Std. & B & Err. Std. & t(16) & p \\
\hline \multirow{2}{*}{ Grande } & Dist. a línea de costa & $-0,381097$ & 0,217931 & $-0,000066$ & 0,000038 & $-1,7487$ & 0,099499 \\
& Altura de la duna & 0,234258 & 0,219843 & 0,001356 & 0,001272 & 1,0656 & 0,302435 \\
& Granulometría & $-0,246337$ & 0,217776 & $-0,009337$ & 0,008255 & $-1,1311$ & 0,274663 \\
\multirow{4}{*}{ Anclitas } & Dist. a línea de costa & $-0,134977$ & 0,252592 & $-0,000020$ & 0,000038 & $-0,5344$ & 0,600436 \\
& Altura de la duna & $-0,107563$ & 0,270271 & $-0,000133$ & 0,000335 & $-0,3980$ & 0,695902 \\
& Granulometría & 0,167152 & 0,258183 & 0,002536 & 0,003917 & 0,6474 & 0,526544 \\
\multirow{4}{*}{ Caguamas } & Dist. a línea de costa & 0,259091 & 0,242234 & 0,000024 & 0,000022 & 1,0696 & 0,300675 \\
& Altura de la duna & $-0,317489$ & 0,239565 & $-0,002479$ & 0,001871 & $-1,3253$ & 0,203695 \\
& Granulometría & $-0,124753$ & 0,237068 & $-0,002953$ & 0,005613 & $-0,5262$ & 0,605947 \\
\hline
\end{tabular}

Tabla 5

Relación de la diversidad funcional (mecanismo de dispersión) con las variables abióticas medidas

\begin{tabular}{llrrrrrc}
\hline Cayos & \multicolumn{1}{c}{ Variables } & \multicolumn{1}{c}{ Beta } & Err Std. & B & Err. Std. & t(16) & p \\
\hline \multirow{2}{*}{ Grande } & Dist. a línea de costa & $-0,382762$ & 0,219713 & $-0,000076$ & 0,000043 & $-1,7421$ & 0,100677 \\
& Altura de la duna & 0,240279 & 0,221640 & 0,001588 & 0,001465 & 1,0841 & 0,294389 \\
& Granulometría & $-0,217179$ & 0,219556 & $-0,009400$ & 0,009503 & $-0,9892$ & 0,337302 \\
\multirow{4}{*}{ Anclitas } & Dist. a línea de costa & $-0,130879$ & 0,253878 & $-0,000020$ & 0,000040 & $-0,5155$ & 0,613239 \\
& Altura de la duna & $-0,136055$ & 0,271647 & $-0,000177$ & 0,000353 & $-0,5009$ & 0,623294 \\
& Granulometría & 0,118448 & 0,259498 & 0,001884 & 0,004127 & 0,4565 & 0,654201 \\
\multirow{5}{*}{ Caguamas } & Dist. a línea de costa & 0,138346 & 0,248204 & 0,000010 & 0,000018 & 0,5574 & 0,584977 \\
& Altura de la duna & $-0,316197$ & 0,245469 & $-0,001901$ & 0,001476 & $-1,2881$ & 0,216018 \\
& Granulometría & $-0,013325$ & 0,242911 & $-0,000243$ & 0,004427 & $-0,0549$ & 0,956932 \\
\hline
\end{tabular}

mero de caméfitas en los cayos Grande y Anclitas, con estrategias de colonización lenta ante perturbaciones, queda explicado por la existencia de una cresta arrecifal, la cual brinda una mayor protección a las costas arenosas ante los embates del viento y penetración del mar. Por otra parte el desarrollo de hemicriptófitas, sobre todo de Distichlis spicata (L.) Greene en Caguamas, se encuentra relacionado con la vulnerabilidad de la costa, debido a la ausencia de una zona de retención de oleaje. Dicha característica concuerda con el desarrollo de plantas herbáceas, donde en dunas incipientes resisten elevada salinidad del substrato y una fuerte dinámica litoral. Sobre este aspecto, un estudio realizado por Álvarez \& Ricardo (en prensa a) en plantas de igual distribución (Sesuvium portulacastrum L. y Paspalum distachyum Poit.), indica la alta capacidad regenerativa de los tallos de estas especies después de haber estado varios días desprendidos del substrato.
El predominio de hojas nanófilas demuestra la adaptación de las especies vegetales a un clima seco. En este sentido, Álvarez \& Ricardo (2009) plantean que el tamaño y estructura funcional de las hojas en las dunas costeras demuestran que la inmensa mayoría de las especies presentan adaptación a las condiciones que impone el invierno seco y ventoso.

En los cayos Grande y Anclitas la zoocoria estuvo determinada por un alto número de plantas con frutos carnosos, destacándose las especies caméfitas y fanerófitas. Por otra parte, el elevado número de plantas anemócoras en cayo Caguamas se establece a partir de la presencia de gramíneas y ciperáceas. Resultados semejantes fueron obtenidos por García (1990) en el Sur de la Isla de la Juventud, donde señala la zoocoria como principal mecanismo de dispersión de árboles y arbustos y la anemocoria para el caso de las hierbas, lianas y epífitas.

La diversidad funcional no varía ante los cambios estacionales, lo cual está determinado por la poca variación 
experimentada por los grupos funcionales, los cuales en su mayoría son especies perennes. Aunque específicamente para la vegetación de costa arenosa no se han usado los índices de diversidad funcional, Álvarez \& Ricardo (en prensa b) hacen alusión a la variación que sufren las formas de vidas terófitas con relación a los períodos de lluvia y seca. Si bien la diversidad funcional muestra pocos cambios en los períodos de seca y lluvia, es probable que estas variaciones sean más evidentes a partir del análisis de la biomasa por especies.

Los factores abióticos medidos no influyen sobre la diversidad funcional en los cayos Grande, Anclitas y Caguamas. Estos resultados se contraponen a los expuestos por Águila et al. (1995), los cuales establecen diferencias entre las asociaciones fitocenólogicas a partir del porte y tamaño de la hoja de las especies vegetales, presente en cada una de ellas. Dichas diferencias están determinadas principalmente por la exposición a los agentes erosivos, los cuales influyen en las características físicas y químicas del substrato. Sobre este aspecto, García-Mora et al. (1999) demuestra que la proporción de los grupos funcionales se encuentra determinada por la dinámica sedimentaria que presenta cada segmento de la duna costera en el Golfo de Cádiz, España.

\section{AGRADECIMIENTOS}

A Dariel López y Eduardo del Sol por la ayuda en el trabajo de campo. Al colega Alain Parada por la revisión del manuscrito. A los evaluadores anónimos por sus acertadas sugerencias.

\section{BIBLIOGRAFÍA}

Acevedo, C. J. \& González-ToRRes, L. R. 2013. Variación espacial de la diversidad en la vegetación de costa arenosa de los cayos Grande, Anclitas y Caguamas, archipiélago de los Jardines de la Reina, Cuba. Mesoamericana 17(1): 9-18.

Acevedo-Rodríguez, P. \& Strong, M. T. 2012. Catalogue of seed plants of the West Indies. Smithson. Contrib. Bot. 98: 1-1193.

Águila, N.; Moreno-Casasola, P.; Menéndez, L.; García, R. \& ChIAPPY, C. 1995. Vegetación de las dunas de Lomas del Puerto (Cayo Coco, Ciego de Ávila, Cuba). Fontqueria 42: 243-256.

Alain, Hno. 1953. Flora de Cuba, 3. Dicotiledóneas: Malpighiaceae-Myrtaceae. Contr. Ocas. Mus. Hist. Nat. Col. La Salle 13. La Habana, Cuba.

Alain, Hno. 1957. Flora de Cuba, 3. Dicotiledóneas: Melastomataceae-Plantiginaceae. Contr. Ocas. Mus. Hist. Nat. Col. La Salle 16. La Habana, Cuba.

Alain, Hno. 1964. Flora de Cuba, 4. Rubiales-ValerianalesCucurbitales-Campanulales-Asterales. Asoc. Estud. Cienc. Biol. La Habana, Cuba.

Álvarez, A. \& RiCARDO, N. 2009. Flora y vegetación de las Playas del Este, Ciudad de La Habana, Cuba I. Flora de las dunas. Acta Bot. Cub. 205:10-25.

Álvarez, A. \& RicARDO, N. (en prensa a). Flora y vegetación de Playas del Este. Ciudad de La Habana, Cuba II. La vegetación de las dunas. Acta Bot. Cub.

ÁlVAREZ, A. \& Ricardo, N. (en prensa b). Flora y vegetación de las dunas de Playas del Este, Cuba III. Vías para la reproducción masiva de plantas para la rehabilitación integral de las dunas. Acta Bot. Cub.

Barbour, M. G.; De Jong, T. M. \& Pavlik, B. M. 1985. Autoecology of marine beach and dune plants on the Atlantic, Gulf and Pacific Coasts of North America. En: B. F. Chabot \& H. A. Mooney (Eds), Physiological ecology of North American plant communities: 296-322. New York.
Blits, K. C. \& Gallagher, J. L. 1991. Morphological and physiological responses to increased salinity in marsh and dune ecotypes of Sporobolus virginicus (L.) Kunth. Oecologia 87(3): 330-335.

BorHIDI, A. 1976. Fundamentos de geobotánica en Cuba. Tesis para el grado de Doctor en Ciencias Biológicas.

Catasús, L. J. 1997. Las gramíneas (Poaceae) de Cuba I. Fontqueria 41: 1-259.

Catasús, L. J. 2002. Las gramíneas (Poaceae) de Cuba II. Cavanillesia altera 3: 1-163.

DíAz, L. R. 1989. Mapa de regionalización climática de Cuba (escala 1: 2000 000). En: ICGC e IG-ACC (Eds.), Nuevo Atlas Nacional de Cuba. Clima, VI. 4.4. Gráficas Alber, España.

GarcíA-AibÁ, J. \& Morey, M.1981. La vegetación de las dunas litorales y su relación con la morfología dunar y el gradiente de influencia marina. Mediterránea 5: 3-22.

García, E. E. 1990. La vegetación del sur de la Isla de la Juventud. Tesis en opción al Grado de Candidato a Doctor en Ciencias Biológicas. Instituto de Ecología y Sistemática.

García-Mora, M. R.; Gallego-Fernández, J. B. \& GarcíaNovo, F. 1999. Plant functional types in coastal foredunes in relation to environmental stress and disturbance. J. Veg. Sci. 10: 27-34.

GISD. 2012. Sporobolus indicus. http://www.issg.org/database/ welcome. Último acceso: 13 de diciembre 2012.

González de Zayas, R.; ZúNiga, A.; Camejo, O.; Batista, L. M. \& CÁrdENAS, R. 2006. Atributos físicos del ecosistema Jardines de la Reina. En: F. Pina (Ed.), Ecosistema costeros: biodiversidad y gestión de los recursos naturales. Compilación por el XV Aniversario del Centro de Investigaciones de Ecosistemas Costeros (CIEC): 296-351. Editorial CUJAE.

HESP, P. 1991. Ecological processes and plant adaptations on coastal dunes. J. Arid. Environ. 21: 165-191.

Kader, G. D. \& PerRY, M. 2007. Variability for categorical variables. J. Stat. Educ.15: 2. 
León, Hno. 1946. Flora de Cuba 1. Gimnospermas, Monocotiledóneas. Contribuciones Ocasionales del Museo de Historia Natural. Colegio «De la Salle». 8. La Habana.

León, Hno. \& Alaín, Hno. 1951. Flora de Cuba 2. Dicotiledóneas: Casuarináceas-Meliáceas. Contribuciones Ocasionales del Museo de Historia Natural. Colegio «De la Salle». 10. La Habana.

Lonard, R. I.; JudD, F. W. \& StaLter, R. 2010. The biological flora of coastal dunes and wetlands: Spartina patens (W. Aiton) G. H. Muhlenberg. J. Coast. Res. 26(5): 935-946.

Martínez, M. L; Valverde, T. \& Moreno-Casasola, P. 1992. Germination response to temperatura, salinity, light and depth of sowing of ten tropical dune species. Oecología 92: 343-353.

Moreno-Casasola, P. 1982. Ecología de la vegetación de dunas costeras: factores físicos. Biótica 7(4): 578-602.

Poos, M. S.; Walker, S. C. \& Jackson, D. A. 2009. Functional-diversity indices can be driven by methodological choices and species richness. Ecology 90(2): 341-347.

RAUNKIAER, C. 1934. The life forms of plant statistical geography. Clarendon Press, Oxford.

RodríGUEZ, A. 2000. Tiliaceae. En: W. Greuter (Ed.), Flora de la República de Cuba. Serie A, plantas vasculares. Fascículo 3(4): 1-38. Koeltz Scientific Books, Königstein.

San Martín, J; Ramírez, C. \& San Martín, C. 1992. La flora de las dunas chilenas y sus adaptaciones morfológicas. Bosque 13(1): 29-39.

Schleuter, D.; Daufresne, M; Massol, F. \& Argillier, C. 2010. A user's guide to functional diversity indices. Ecological Monographs 80(3): 469-484.
Shannon, C. E. \& Weaver, W. 1949. The mathematical theory of communication. Urbana Univ. Illinois Press. USA.

Simpson, E. H. 1949. Measurement of diversity. Nature 163: 688.

SkARADEK, W. \& Miller, C. 2010. Saltgrass Distichlis spicata (L.) Greene Plant Symbol = Disp. USDA-Natural Resources Conservation Service. www.plant-materials.nrcs. usda.gov/...etpmcfs 77 pdf. Último acceso: 13 de diciembre 2012.

StatsofT, Inc. 2001. Statistica (data analysis software system), version 6. www. statsoft.com. Último acceso: 13 de diciembre 2012.

TikU, B.L. \& SNaYdon, R. W. 1971. Salinity tolerance within the grass species Agrostis stolonifera L. Plant and Soil 35: 421-431.

VAND DER PIJL, L. 1982. Principles of dispersal in higher plants. 3ra Edición. Springer-Verlag, NewYork, USA.

WiLLIAMs, M. J. 2007. Native plants for coastal restoration: what, when, and how for Florida. USDA, NRCS, Brooksville Plant Materials Center, Brooksville, FL. 51pp. http://www.fl.nrcs.usda.gov/programs/pmc/flplantmaterials.html. Último acceso: 13 de diciembre 2012.

WoodeLL, S. R. J. 1985. Salinity and seed germination patterns in coastal plants. Vegetatio 61: 223-229.

ZoNA, S. 1990. A monograph of Sabal (Arecaceae: Coryphoideae). Aliso 12(4): 583-666.

ZÚÑIGA, A. 2000. Caracterización básica de la geología de los cayos de la porción centro occidental del subarchipiélago Jardines de la Reina. Cayos Algodón Grande, Anclitas y Caballones. Enlace 6(33): 1-5. 